\title{
Unità e pluralità delle scienze sociali
}

\section{Vittorio Cotesta}

Università degli Studi "Roma Tre», Dipartimento di Scienze della Formazione

doi: 10.7358/ecps-2014-009-cote

vittorio.cotesta@uniroma3.it

\section{UNITY AND PLURALITY OF THE SOCIAL SCIENCES}

\begin{abstract}
The author supports the view that New Realism is a positive reaction to the worst aspects of Post-modernism. The call to reality and realism is also a recurrent theme in the scientific debate, both when new sciences are founded and also in periods of paradigm change. This view is demonstrated through two cases - Thucydides criticism of Herodotus at the time of the foundation of history as a science, and Durkheim's call to reality in the "Rules of the sociological method". However, the author maintains that this call to reality is accompanied by a new vision of the social world, and this is seen better in the methodological works of $M$. Weber. By analyzing these cases, the author presents the main thesis that sciences - all empirical sciences and not just the social sciences - are such if, and only if, their logical structure is based on three principles: (a) theoretical (internal) coherence, (b) correspondence of the theory with the empirical world, and (c) liberty, autonomy, and responsibility of the scientists. All sciences, and not just the empirical ones, are instead differentiated from one another by their object andlor by the way they view their object. This general view is illustrated through a case study of the social sciences and particularly by analyzing the epistemological structure of sociology.
\end{abstract}

Keywords: Durkheim, Methodology, Paradigm, Social sciences, Weber.

Se Pirro non fosse caduto ad Argo per mano di una brutta vecchiaccia, o Giulio Cesare non fosse stato ucciso a coltellate. Cose che non si possono cancellare con il pensiero. Il tempo li ha segnati con il suo marchio, e in ceppi dimorano nel luogo delle infinite possibilità che esse hanno estromesso. Ma possono essere stati possibili dato che non furono mai? $\mathrm{O}$ fu possibile solo ciò che avvenne?

Joyce, Ulisse (1973, p. 25)

ECPS Journal - 9/2014

http://www.ledonline.it/ECPS-Journal/ 


\section{1. «NEW REALISM»?}

Da alcuni decenni le scienze sociali vivono una crisi profonda. Le "grandi narrazioni» del mondo da loro elaborate nel corso del XIX e XX secolo sono diventate quasi di colpo inservibili. Molti, per superare la crisi, hanno cercato una via d'uscita dedicandosi allo studio di nuovi temi emergenti come la «micro-sociologia», le pratiche della vita quotidiana, gli studi di genere, le migrazioni, i nuovi media e i loro effetti. Per usare un concetto caro ad Alfred Schütz (1971, p. 203), la sociologia si è configurata come una scienza di "province finite di significato" senza più tentare grandi analisi capaci di elaborare una teoria del mondo.

Naturalmente: non tutto è negativo in questo movimento di rifiuto di teorie il più delle volte espressioni delle grandi narrazioni ottocentesche, divenute ormai dei puri fossili ideologici. Quel che inquieta, però, è l'atteggiamento rinunciatario, come se, esaurita l'efficacia di quelle concezioni, non si potessero cercare altre vie per elaborare nuove teorie del mondo.

Si tratta, in verità, di una percezione unilaterale della situazione storica nella quale viviamo. Teorie generali del mondo in realtà sono state elaborate proprio nel pieno di questa crisi e su di esse verte ora il dibattito teorico e metodologico. Inoltre, essa rifletteva - e riflette - la crisi del mondo occidentale nel nuovo contesto mondiale. Nei paesi emergenti (ad esempio, Cina, India, Sud Africa ed America Latina) non si vede una crisi delle scienze sociali. Da un lato i sociologici sono impegnati nella critica e denuncia del punto di vista "eurocentrico", dall'altro si connettono ai movimenti sociali dei loro paesi oppure ai tentativi dei loro Stati di trovare una via di sviluppo in parte diversa dalla way of life occidentale.

Qualcosa di analogo è avvenuto in filosofia. Finita anche qui la stagione delle grandi narrazioni, è emerso un atteggiamento decostruzionista. Pure qui sono prevalse analisi di «regioni finite» della vita, la scomposizione delle visioni del mondo, i discorsi micro, spesso suggestivi ma programmaticamente votati alla negazione della possibilità di tratteggiare una nuova teoria del mondo. A tutto questo movimento è stato dato, con molta approssimazione, il nome di post-moderno.

$\grave{E}$ una fatica dura contestare le mode. Ma, volgendo ora lo sguardo indietro, non si può non vedere come proprio in questi decenni sia stata elaborata una nuova visione della modernità. L'acquisizione più importante in questo campo è che non vi sia stata una sola modernità, ma molteplici, ognuna con caratteri simili e diversi dalle altre (Eisenstadt, 2003, pp. 519-560).

In filosofia, inoltre, è emerso un fastidio per i discorsi sui discorsi e sulle decostruzioni. Il New Realism è un richiamo a porre il mondo al centro dell'analisi filosofica. 
Un brusco richiamo alla realtà, come quello proposto dal New Realism, è solo in parte un fatto nuovo. Esso è nuovo per il contesto nel quale viene proposto; è un fenomeno ricorrente, invece, per la forma che assume.

Si tratta infatti dell'espressione di tendenze profonde nelle scienze umane e sociali ed emergono nei periodi di fondazione delle civiltà oppure nei momenti di svolta e rivoluzione dei fondamenti stessi delle società. Nelle pagine che seguono tenteremo di darne degli esempi.

\section{FATTI E TEORIA E NELLE SCIENZE SOCIALI}

Nella nostra tradizione culturale il conflitto metodologico tra ciò che abbiamo imparato a chiamare "positivismo" e «idealismo" risale al tempo di Erodoto e di Tucidide, di Platone e Aristotele, almeno. Da un lato i «fatti», le forme concrete della vita, dall'altro le idee (eidos), le immagini, le visioni. Che ci fosse - e ci sia - un rapporto di reciproca interdipendenza tra le due sfere - la vita e le forme - raramente arriva alla consapevolezza metodologica.

Può essere interessante, infatti, vedere come l'opposizione positivismoidealismo si configura nel momento della fondazione delle scienze storiche in Grecia ${ }^{1}$. Vediamo come si presenta questa contrapposizione in uno dei momenti non solo della fondazione delle scienze storiche ma della stessa costruzione del punto di vista "greco" sul mondo. In realtà, Erodoto e Tucidide, con le loro storie non stanno lavorando solo alla narrazione di grandi avvenimenti del mondo antico (il rapporto con i persiani, Erodoto; il rapporto tra i greci, Tucidide), ma stanno gettando le basi della costruzione dell'identità greca e, per i risvolti storici successivi, del mondo occidentale. Con parole di sintesi si potrebbe esprimere il tutto così: Erodoto costruisce la narrazione dei rapporti dei greci con l'Altro; Tucidide affronta, invece, la narrazione dei rapporti dei greci con se stessi. Le loro storie sono alla fine un capitolo della costruzione dell'identità greca. Perciò le questioni metodologiche sono di grandissima rilevanza. Quali fatti «raccontare», come raccontarli, quali prove si hanno che i fatti rilevanti sono questi e non altri. Quali sono le prove incontestabili che gli eventi si sono svolti in questo modo e non altrimenti? Chi è un testimone degno di fede e chi no? Queste sono solo alcune delle

1 Se facciamo riferimento ad Erodoto e Tucidide non è perché ignoriamo l'esistenza di altri storici del mondo greco ma perché Tucidide rivolge ad Erodoto aspre critiche per il suo approccio allo studio della storia. Una pretesa di descrivere i puri fatti (Tucidide) contro una prassi della ricerca storica più ampia, oggi potremmo chiamarla «multidimensionale». 
questioni che essi devono risolvere. Ma partiamo da Tucidide, che si esprime molto severamente verso Erodoto.

Dopo aver narrato la «preistoria» della Grecia, Tucidide afferma:

Tali dunque si sono presentati alle mie ricerche gli antichi avvenimenti, ma sono tali da render difficile il prestar fede a un qualunque indizio su di loro, così come viene. Giacché gli uomini accettano ugualmente senza sottoporle a prova le tradizioni orali degli avvenimenti precedenti, anche se esse riguardano avvenimenti del loro paese [...]. Così poco faticosa è per i più la ricerca della verità, e a tal punto i più si volgono di preferenza a ciò che è a portata di mano. (Tucidide, 1998, I, par. 20)

Il primo criterio metodologico sembra dunque essere per Tucidide diffidare delle versioni correnti e popolari dei fatti. Prima di accoglierli nel proprio discorso è bene sottoporre «le tradizioni orali» a critica. E continua:

Tuttavia, chi, basandosi sulle testimonianze che ho dato, intende in tal modo gli avvenimenti da me esposti e non presta maggior fede alle celebrazioni che i poeti hanno fatto di quegli stessi avvenimenti, abbellendoli, o alle narrazioni dei logografi, aventi per scopo più il diletto dell'udito che la verità, avvenimenti non provabili e per la maggior parte, per effetto del tempo trascorso, passati a far parte del mito in modo da non meritare attendibilità; chi infine crede che tali avvenimenti sono stati investigati sulla base degli indizi più sicuri, in modo sufficiente, data la loro antichità - costui non dovrebbe sbagliare. E questa guerra, sebbene gli uomini considerino più importante sempre la guerra presente a cui partecipano, mentre, una volta finita, ammirano soprattutto gli avvenimenti passati, a giudicare sulla base dei fatti stessi si vedrà che è stata maggiore di quelle del passato. (ivi, par. 21)

Qui la distanza critica suggerita riguarda il mito e l'atteggiamento verso il passato. La storia non può prendere come fatti veri quelli narrati nei miti. Occorre inoltre liberarsi da due atteggiamenti opposti: sopravvalutare il presente e il passato ma "giudicare solo sulla base dei fatti».

Il punto seguente è invece di grande rilevanza epistemologica, come vedremo, ancora oggi.

E quanto ai discorsi che tennero gli uni e gli altri sia in procinto di far la guerra che durante, tenere a mente le parole precise di quei discorsi era difficile tanto per me, nel caso in cui le avessi udite personalmente, quanto per quelli che me le riferivano da qualche altro posto; ma a seconda di quanto ciascuno a mio parere avrebbe potuto dire nel modo più adatto nelle diverse situazioni successive, così si parlerà nella mia opera, ché io mi terrò il più possibile vicino al pensiero generale dei discorsi effettivamente pronunciati. (ivi, par. 22) 
Come si sa, ognuno dei combattenti deve legittimare a se stesso, ai propri uomini e, per quanto possa apparire paradossale, anche ai propri nemici la guerra che combatte. Perciò, non avendo i «documenti» da cui ricavare i discorsi tenuti dagli attori in guerra, occorre immaginare - lo diciamo con le parole di M. Weber - una situazione tipico-ideale. Tucidide, cioè, immagina cosa avrebbe detto l'uno e l'altro combattente in una situazione tipica: «a seconda di quanto ciascuno a mio parere avrebbe potuto dire nel modo più adatto nelle diverse situazioni», e su questa base ricostruisce i discorsi dell'uno e dell'altro dei personaggi della sua storia.

Infine, la prova:

I fatti concreti degli avvenimenti di guerra non ho considerato opportuno raccontarli informandomi dal primo che capitava, né come pareva a me, ma ho raccontato quelli a cui io stesso fui presente [Tucidide ha partecipato alla Guerra del Peloponneso] e su ciascuno dei quali mi informai dagli altri con la maggior esattezza possibile. Difficile era la ricerca, perché quelli che partecipavano ai fatti non dicevano tutti le stesse cose sugli avvenimenti stessi, ma parlavano a seconda del loro ricordo o della loro simpatia per una delle due parti. La mancanza del favoloso in questi fatti li farà apparire, forse, meno piacevoli all'ascolto, ma se quelli che vorranno investigare la realtà degli avvenimenti passati e quelli futuri (i quali, secondo il carattere dell'uomo, saranno uguali o simili a questi), considereranno utile la mia opera, tanto basta. Essa è un possesso che vale per l'eternità più che un pezzo di bravura, da essere ascoltato momentaneamente. (ibid.)

Tucidide vaglia le prove. Egli stesso è un testimone, ma non basta. Occorre informarsi dagli altri «con maggiore esattezza possibile», e considerare che il ricordo può distorcere la verità dei fatti e la simpatia per l'uno o per l'altro dei contendenti può giocare brutti scherzi.

In sintesi, dunque, Tucidide mette l'accento sulla necessità di vagliare criticamente i fatti, le loro diverse narrazioni, tener conto delle distorsioni dovute alle «simpatie» per l'una o per l'altra parte. Rimane inoppugnabile, però, che per lui lo storico deve «descrivere», «narrare» $\mathrm{i}$ «fatti». Diverso infatti è l'approccio dello storico da quello dei poeti e dei logografi. Inoltre, occorre vagliare criticamente le fonti: «I fatti concreti degli avvenimenti di guerra non ho considerato opportuno raccontarli informandomi dal primo che capitava, né come pareva a me, ma ho raccontato quelli a cui io stesso fui presente e su ciascuno dei quali mi informai dagli altri con la maggior esattezza possibile».

Anche lui però alla fine è costretto a fare della storia un "racconto", una «narrazione». Perciò, dovendo far parlare i diversi soggetti o attori delle vicende che racconta, costruisce una situazione tipica e fa dire ad ognuno ciò che in quella situazione (tipica) è ragionevole che si dica. 
Erodoto aveva seguito una via analoga ma più complessa. Oltre ai fatti, alle esperienze personali - Erodoto è stato un grande viaggiatore - egli ritiene che ciò che gli uomini dicono, sia verità o mito, è utile alla comprensione degli avvenimenti. Contrariamente a quanto sostengono i suoi detrattori, Erodoto non crede ad ogni cosa, ma vaglia criticamente e comunque riporta le opinioni dei suoi informatori: "Io sono tenuto a riferire quel che si dice, ma non a prestare fede a tutto e queste parole valgano per ogni mia trattazione» (Erodoto, 2000, VII, 152, par. 3). Il mito può essere utile per comprendere la storia, secondo Erodoto. Per noi è come dire che abbiamo bisogno di sapere cosa pensano gli attori sociali per comprenderne le azioni.

Il richiamo critico di Tucidide ai «fatti» è il topos del nostro interesse. Si può osservare, tuttavia, che il richiamo ai fatti non evita la necessità dell'elaborazione di idee che rendono significativi quei fatti medesimi. Insomma, per «spiegare» gli avvenimenti si devono dare «ragioni» delle loro connessioni logiche, altrimenti essi non avrebbero nessun significato. Non si può fare "scienza» senza idee, concetti e teorie. Perfino Tucidide è costretto a farlo.

L'altro esempio di richiamo ai fatti è più vicino a noi. Per intendere bene il senso del discorso occorre considerare che ci troviamo in una situazione tipica, di fondazione di una nuova scienza, che deve prendere le distanze da concezioni e pratiche correnti giudicate erronee.

$\mathrm{Ne}$ Les règles de la méthode sociologique, capitolo II, Émile Durkheim esordisce scagliando un pugno nell'occhio ai suoi avversari: «La prima regola - che è anche la più fondamentale - impone di considerare i fatti sociali come cose» (Durkheim, 1895, p. 35).

È difficile, oggi, capire a chi si rivolgesse concretamente Durkheim. Forse a Comte, suo "maestro», dal quale si allontanava perché non ne condivideva la filosofia positivista della storia; oppure a Herbert Spencer, comunque apprezzato e, nello stesso tempo criticato, nelle sue opere. $\mathrm{O}$ forse anche - è una mia supposizione malevola - ai «tedeschi» che non nomina mai, come Max Weber, oppure come Georg Simmel, che nomina ma critica aspramente. Chi può dire?

Sembrerebbe, stando alle dichiarazioni formali di Durkheim, che la sociologia sia una scienza fondata sull'osservazione dei fatti, che, per loro natura, non sono diversi dalle cose studiate dalle scienze naturali. Osservare, rilevare, misurare, stabilire correlazioni. Bene. Molto bene. E dopo?

Dopo comincia il lavoro difficile. Occorre stabilire delle relazioni «astratte», guarda un po'!?, costruire dei tipi, fare ipotesi e verificare se tengono.

Se uno prendesse per buone le dichiarazioni - queste dichiarazioni - di Durkheim, dovrebbe arrendersi di fronte ad un positivismo piuttosto rude. $\mathrm{Ma}$, sia detto senza cattiveria, per apprezzare Durkheim, occorre liberarlo dal suo linguaggio, dalle sue metafore prese in prestito dalla filosofia orga- 
nicistica e dalla meccanica (si pensi ai suoi concetti chiave di «solidarietà meccanica» e di «solidarietà organica», giusto per fare un esempio) ${ }^{2}$. Su un punto, però, occorre restare fermi: i fatti sociali di cui si occupa la sociologia, che sono "cose» solo nella metafora, sono «esterni» all'individuo e, anche, allo studioso. Insomma, occorre partire dal sano principio kantiano secondo cui la realtà di cui ci occupiamo è fuori di noi. Non possiamo pensare di risolverla, à la Berkeley, nella nostra percezione. Anche quando si tratta di idee, emozioni e sensazioni, per poterle studiare, dobbiamo considerarle come esistenti indipendentemente da noi. E questo vuole dire Durkheim. Ciò di cui si occupa la sociologia sono i fatti sociali esistenti nella realtà e possono essere spiegati solo da altri fatti sociali, non da fattori psicologici e neppure $\mathrm{da}$ fattori economici. Non si può ridurre l'individuo ad una marionetta, come fa la teoria marginalistica, e neppure, come vorrebbe la psicologia, sempre pronta a rintracciare nella psiche i fattori che determinano le azioni degli individui. Il suicidio è, da questo punto di vista, la prima grande prova che comportamenti «individuali» sono determinati dalle relazioni sociali. L'essere con e per gli altri costituisce l'orizzonte dell'azione degli individui. E si agisce con, per e contro gli altri. Ciò non significa che non possano esistere altre dimensioni, come quella biologica o quella psicologica. Vuol dire solo che esse costituiscono il contesto pre-sociale sul quale operano i fattori sociali; e, naturalmente, vi è la relazione inversa.

Il punto più forte della posizione di Durkheim - punto forte ancora oggi - è il suo modo di intendere la sociologia come scienza della comparazione: "la sociologia comparata è la sociologia stessa» (Durkheim, 1895, p. 126). Da un lato il metodo comparativo consiste nella costruzione di tipi puri, per utilizzare un lessico weberiano non estraneo a Durkheim (ivi, p. $84^{3}$ ), e dall'altro nella comparazione tra i diversi tipi o casi, costruiti per via teorica e concettuale: «[...] dopo aver stabilita la classificazione in base a questo principio [cfr. nota 3], per sapere se un fatto sia generale in una specie

2 Un'operazione analoga compie Boudon (1994) sul concetto di magia in Durkheim. Credo, però, che la stessa operazione si possa fare anche con Les règles de la méthode sociologique (1895).

Poiché questo a me sembra un punto molto importante del ragionamento, mi pare corretto dare il passo completo a cui mi riferisco. Nel capitolo «Regole relative alla costituzione dei tipi sociali» Durkheim afferma: la classificazione «sarà veramente utile soltanto se ci permetterà di classificare caratteri differenti da quelli che le servono da base - cioè se ci procurerà quadri concettuali per i fatti futuri. Essa ha la funzione di fornirci punti di riferimento ai quali si possano collegare osservazioni diverse da quelle che ci hanno fornito questi stessi punti di riferimento. Ma a tale scopo occorre che essa venga stabilita non già in base ad un inventario completo di tutti i caratteri individuali, bensi in base ad un piccolo numero di essi, accuratamente scelti [...]. Dobbiamo quindi scegliere per la nostra classificazione dei caratteri particolarmente essenziali» (Durkheim, 1895, p. 84; corsivo mio). 
non sarà necessario osservare tutte le società di quella specie; alcune di esse basteranno. Anzi, in molti casi un'osservazione ben fatta sarà sufficiente, così come un'esperienza ben fatta basta spesso per stabilire una legge» (ibid.).

Il caso di Max Weber è a mio avviso ancora più interessante. La sua pratica della sociologia si svolge dentro il dibattito sulla natura delle scienze storico-sociali, se esse possano essere - come sembra ritenere Durkheim - come le scienze della natura o se debbano essere differenti (il cosiddetto primo Methodenstreit ${ }^{4}$.

La prima questione è se Durkheim, con il suo motto: «considerare i fatti sociali come cose», voglia o possa colpire Max Weber. Weber da questo punto di vista è un kantiano, come Durkheim. Un po' più sofisticato, però. Egli lavora alla costruzione della sociologia come una scienza "empirica», che si occupa quindi di fatti esistenti nel mondo e non solo nella testa del ricercatore. «La scienza sociale, quale noi vogliamo promuoverla - scrive Weber -, è una scienza di realtà. Noi vogliamo intendere la realtà della vita che ci circonda, e nella quale noi siamo inseriti, nel suo proprio carattere - noi vogliamo intendere cioè da un lato la connessione e il significato culturale dei suoi fenomeni particolari nella loro odierna configurazione, e dall'altro i fondamenti del suo essere storicamente divenuto così-e-non-altrimenti» (Weber, 1904, p. 84). Il problema è come vengono intesi i fatti sociali e, soprattutto, come debbano essere spiegati e interpretati. Il punto di massima rilevanza è che Weber pone al centro della sua costruzione teorica quale unità analitica elementare l'agire o la relazione sociale, l'essere con e per gli altri. Nonostante una vulgata abbia interpretato la sua posizione come «individualismo sociologico», egli non pone affatto a base del discorso sociologico l'individuo ma gli individui nella loro reciproca relazione ${ }^{5}:$ «[...] per 'agire sociale' si deve intendere un atteggiamento umano (sia esso un fare o un tralasciare o un subire, di carattere esterno o interno), se e in quanto l'individuo che agisce o gli individui che agiscono congiungono ad esso un senso soggettivo. Per agire 'sociale' si deve però intendere un agire che sia riferito - secondo il suo senso, intenzionato dall'agente o dagli agenti - all'atteggiamento di altri individui, e orientato nel suo corso in base ad esso" (Weber, 1922, p. 4).

Non posso e non voglio dilungarmi oltre nell'illustrare la concezione della sociologia di Max Weber. I trattati su cosa sia o non sia la sociologia

4 Per quanto riguarda il primo Methodenstreit cfr. Bianco, 1978; per quanto riguarda il cosiddetto secondo Methodenstreit cfr. Adorno, Popper et al., 1969.

5 Su questo la posizione di Weber e quella di Simmel convergono: l'unità analitica elementare è l'azione o la relazione sociale. Questa inoltre è intesa non come un mero riferirsi a, ma come un atto che abbia effetti sul comportamento dell'altro o degli altri. Come è noto, Simmel, per esprimere questo complesso di significati dell'azione sociale usa il termine di Wechselwirkung, ove risaltano la reciprocità (Wechsel) e l'efficacia (Wirken) (Simmel, 1908, pp. 8-9). 
di Weber sono infiniti. Voglio però affrontare solo un punto a mio avviso nevralgico per comprendere la portata dell'opera di Max Weber.

Nel quasi secolo trascorso dopo la sua morte (1920) la fortuna di Weber è stata piuttosto alterna: ora sono state apprezzate soprattutto le opere metodologiche, ora Economia e società. I Saggi sulla sociologia della religione raramente sono stati considerati la sua opera più importante, se non forse, tra $\mathrm{i}$ sociologi della religione.

Dal mio punto di vista - e senza togliere nulla all'importanza dei saggi metodologici e all'insieme delle elaborazioni contenute in Economia e societàl'opera più attuale di Weber è proprio La sociologia della religione. Questa mia convinzione è fondata su due tipi di ragioni: una metodologica; l'altra teorica.

L'opera di Weber è soprattutto - come in fondo la sociologia di Durkheim - una sociologia comparativa. La costruzione dei tipi ideali in Weber serve per quella che lui chiama «imputazione causale». Com'è noto - e come sappiamo anche da Durkheim - i tipi ideali sono tipi puri, costruiti concettualmente a partire da alcuni tratti della realtà. Non sono l'inventario completo o una descrizione esaustiva della realtà sociale che si vuole studiare, ma una costruzione basata su alcuni caratteri «essenziali» (Durkheim) di quella realtà. I tipi servono per la comparazione e per la spiegazione causale ${ }^{6}$. La parte più innovativa della posizione di Weber dal punto di vista metodologico è il superamento della dicotomia tra scienze della natura e scienze della cultura o dello spirito. Le prime volte a formulare leggi generali sul mondo; le altre invece tese a comprendere il caso singolo. Weber segue una via diversa: per lui le leggi generali servono per comprendere il caso singolo e - questo per me è il punto di svolta - il caso singolo può essere compreso solo se comparato al tipo puro, che rappresenta la classe o la famiglia dei casi a cui esso appartiene. Insomma, non c'è più contrapposizione ma le leggi generali, stabilite nella ricerca precedente, le uniformità rilevate nel passato, sono tutti mezzi per comprendere il singolo caso attuale (Weber 1904, pp. 85-89, 9394). Inoltre, questo caso appartiene ad una famiglia di casi, e le sue particolarità possono essere comprese solo a confronto con la classe o la famiglia dei casi a cui appartiene. Infine - e su questo tornerò -, la sociologia appartiene alla famiglia delle scienze per la sua logica; alla famiglia delle scienze sociali per il suo oggetto. Nessuna propensione per il «rivivere» diltheyano o per

6 Avverto che sia Weber che Durkheim lavoravano con un concetto di causa preso dalle scienze del XIX secolo e mentre loro stavano rinnovando le scienze sociali, la stessa cosa avveniva nelle scienze fisiche e biologiche. Pertanto, taluni concetti - come quello di causa - presi dalla precedente configurazione delle scienze fisiche, in queste scienze non valevano già più o erano concepiti in modo diverso. Insomma, erano contemporanei di Einstein ma non della fisica prodotta da lui. Sui limiti della concezione del concetto di causa in M. Weber cfr. Cavalli, 2012, pp. 113-131. 
le varie e diverse forme di filosofia della storia, à la Spengler, per esempio. Negli ultimi decenni - e così veniamo alla ragione teorica - proprio mentre imperversava ogni forma di post-modernismo l'opera di Weber - I saggi sulla sociologia della religione - è stata (ed è ancora) al centro del dibattito sulla "modernizzazione». In questo campo di studi l'opera di Weber è stata discussa, criticata, accettata o anche rifiutata. In generale a Weber viene rimproverato (a) di avere un punto di vista eurocentrico; (b) di non aver compreso né il confucianesimo né l'islam; di aver per conseguenza (c) stabilito una relazione fallace tra confucianesimo e mancata modernizzazione della Cina; (d) di aver usato la comparazione tra l'Occidente e la Cina o l'India solo per giustificare la ricerca della singolarità o dell'eccezionalità dell'Occidente. Gli sono poi rimproverate tante altre cose particolari ma sono a mio avviso tutte riconducibili a queste obiezioni più generali. Una critica, in particolare, quella di J. Goody, famoso antropologo inglese, oltre a tutti questi aspetti tratta le questioni metodologiche. Secondo Goody, Weber non doveva ricercare la singolarità ma ciò che è comune alle diverse civiltà. Solo così avrebbe potuto capire che l'industrializzazione e il capitalismo moderno, nati in Europa, potevano ben nascere in Cina che, fino al XVIII secolo, era avanti all'Europa in tutte le aree della società (Goody, 1996 e 2006).

Questa critica misconosce i fondamenti della sociologia comparativa di Weber. Infatti, come abbiamo detto sopra, la comprensione del singolo caso implica l'elaborazione del concetto generale, il tipo puro, a cui il caso appartiene. Weber ha elaborato un concetto multidimensionale della società moderna, tuttora valido, a mio avviso, e sulla base delle dimensioni della teoria ha messo a confronto la società cinese e quella europea. Dalla comparazione non emerge solo la singolarità dell'Occidente ma anche quella della Cina. L'una è il rovescio dell'altra nel senso che, nel comprendere i tratti dell'una, comprendo anche i tratti dell'altra ${ }^{7}$. Il passaggio esplicativo successivo, l'imputazione causale dei fattori che hanno favorito o impedito lo sviluppo della modernità, è sotto osservazione critica e non da oggi. Sono tanti infatti i dubbi sul nesso causale tra etica protestante e spirito del capitalismo così come quelli sull'etica confuciana quale impedimento per la nascita del capitalismo moderno in Cina. Rimane, però, la lezione metodologica weberiana. Nella sociologia vi è un cambiamento di paradigma, indotto dalla diffusa consapevolezza di vivere in una società globale e, in questo campo di studi, la sociologia comparativa di Weber è un esempio ancora molto valido. Non per nulla, l'inventore della teoria delle modernità multiple, S.N. Eisenstadt, vede la sua teoria come una riforma e non come un superamento delle posizioni di M. Weber (Eisenstadt, 1997).

7 Sulla comparazione in Durkheim e Weber, sulla loro convergenza divergenza cfr. Smelser, 1976, in modo particolare le pp. 205-206. 


\section{UNITÀ E PLURALITÀ DELLE SCIENZE SOCIALI ${ }^{8}$}

Nelle pagine precedenti si trovano qua e là osservazioni e affermazioni che ora è il caso di rendere sistematiche per sostenere la tesi di questo articolo. La scienza, secondo la sua logica, è una; secondo il suo contenuto, cioè l'oggetto di cui si occupa, è plurale.

\subsection{La logica scientifica è una e una sola}

Per poter legittimare questa affermazione è necessario fare un lungo ragionamento sulla logica e sulla metodologica scientifica. Dobbiamo distinguere in primo luogo il metodo della conoscenza dalla realtà che si vuole conoscere. In questo paragrafo ci occuperemo solo del metodo.

Nella lingua greca, originariamente, metodo significa via (hodòs), percorso. Perciò, per metodo dobbiamo intendere la via della conoscenza.

Queste affermazioni, però, sono del tutto preliminari. Infatti, occorre domandarsi subito: quale via porta alla conoscenza? Quale percorso ragionevolmente può condurci a conoscere qualcosa del mondo in cui viviamo? Rispondere a queste domande implica risolvere una serie di problemi che nella storia sono stati pensati come il problema della verità dei discorsi, delle teorie e delle leggi scientifiche. La prima e fondamentale regola è stata, almeno nella tradizione che va da Aristotele fino a Popper, la seguente: rifiutare tutte le proposizioni contraddittorie; quelle proposizioni che, nello stesso tempo e luogo, affermano che $\mathrm{A}=-\mathrm{A}$. Se qualcosa appare contraddittoria, afferma Popper, occorre lavorare per trovare ove si annida il nostro errore ed eliminarlo (Popper 1969a, p. 539). Se poi altri, come è nella tradizione che va da Eraclito fino a Hegel, ritengono che la realtà sia intrinsecamente contraddittoria, si può rispondere con le parole di Aristotele: solo per «incultura» si può ritenere che una cosa sia nello stesso tempo uguale e diversa da se stessa (Aristotele, 1994, 1006 b, 5-10). Dunque, nell'elaborazione di un metodo conoscitivo, dobbiamo partire dall'opzione in favore del principio di identità: $p=p$ ed è impossibile che $p=\sim p$.

A livello di singole proposizioni quest'opzione in favore dell'identità è piuttosto facile, anche se non bisogna mai dimenticare che il linguaggio è

8 Sull'unità e pluralità delle scienze sociali ha riflettuto pure Berthelot (2001, pp. 456519). La mia riflessione ha tuttavia un obiettivo completamente diverso; diversa è pura la base bibliografica e il contesto di riferimento. Berthelot si muove nell'ambito francese; per me come si può constatare - l'ambito di riferimento è costituito dalle scienze sociali, se così si può dire, «europee». 
opaco, ambiguo e fuorviante. Il problema diventa invece molto complicato nell'elaborazione di grandi teorie sul mondo. Perciò l'analisi interna alle catene di proposizioni nelle quali consistono le teorie diventa fondamentale per non arrivare a conclusioni ambigue e contraddittorie. Insomma, tra le diverse proposizioni di un discorso o di una teoria $\mathrm{T}$, ci deve essere coerenza. Detto altrimenti, se $\mathrm{T}=\left(\mathrm{p}_{1}, \mathrm{p}_{2}, \mathrm{p}_{3}, \ldots \mathrm{p}_{\mathrm{n}}\right)$, nessuna proposizione dell'insieme $\left\{\mathrm{p}_{1}\right.$, $\left.\mathrm{p}_{2}, \mathrm{p}_{3}, \ldots \mathrm{p}_{\mathrm{n}}\right\}$ deve contraddire un altro membro dell'insieme. Se una teoria soddisfa questo requisito logico, si può dire che sia coerente o vera.

Per le scienze empiriche tutto questo non basta. Le loro teorie devono soddisfare altri criteri di verità. Per una teoria empirica, che vuole cioè dire qualcosa sul mondo, occorre che essa dia una spiegazione di come il mondo è fatto, sia relativamente alla sua struttura attuale, sia relativamente alla sua costituzione genetica. Quando una teoria T è considerata vera?

$\mathrm{Su}$ questo punto non ci sono posizioni convergenti. Possiamo richiamarne due, la teoria della verità di Popper e quella di Tarski. La teoria di Popper è molto semplice. Essa afferma che tra due teorie, $T_{1}$ e $T_{2}$, è preferibile quella che, nello stesso tempo, contiene una maggiore contenuto di verità e un minore contenuto di falsità. $\mathrm{T}_{2}$ è preferibile a $\mathrm{T}_{1}$ se, e solo se, Ctv $\left(\mathrm{T}_{2}\right)>$ $\operatorname{Ctv}\left(\mathrm{T}_{1}\right)$ e $\operatorname{Ctf}\left(\mathrm{T}_{2}\right)<\operatorname{Ctf}\left(\mathrm{T}_{1}\right)$ (Popper, 1969b, p. 401; cfr. pure Popper, 1934).

La soluzione di Tarsi vuole rimuovere le difficoltà della teoria della verità come corrispondenza. Nella posizione popperiana, del resto, si dà per risolto il problema che si deve risolvere. Decidere della preferibilità di una teoria rispetto ad un'altra sulla base del suo contenuto di verità e di falsità, significa che già si conosca il contenuto di verità o di falsità della teoria. Ma è proprio su questo che occorre avere un criterio in base al quale una teoria è vera e un'altra falsa. Per risolvere questo problema Tarski inventa una serie di tecniche logiche e linguistiche (Tarski, 1943-1944). Il senso della sua teoria della verità come corrispondenza è che una proposizione è vera se, e solo se, in un mondo possibile, ricorre che $\mathrm{p}=\mathrm{v}$. La teoria tarskiana della verità è fondata sul soddisfacimento di una funzione da parte di una proposizione: $\mathrm{f}(\mathrm{p})=\mathrm{x}$. A questo occorre inoltre aggiungere che il soddisfacimento di una funzione può avvenire solo entro un mondo possibile $\mathrm{M}$ oppure in un linguaggio L. Di fatto, la teoria di Tarski ci dice che, se entro un mondo possibile $\mathrm{M}$, i parlanti un linguaggio $\mathrm{L}$, ritengono che una proposizione $\mathrm{p}$ soddisfi la funzione $\mathrm{v}$, allora $\mathrm{p}=\mathrm{v}$, ovvero la proposizione $\mathrm{p}$ è vera.

Come molti casi della storia della scienza dimostrano, però, questa definizione della verità fondata sul consenso della comunità scientifica non dimostra la superiorità di una teoria rispetto ad un'altra ${ }^{9}$. Dimostra solo che, quando in

9 Vedi l'esempio classico sul carattere emissivo o ondulatorio della luce illustrato da P. Duhem (1914) tra Biot e Fresnel. Le pressioni su Biot perché abbandonasse la teoria emissiva 
una comunità scientifica si forma il consenso in una direzione, coloro che non sono d'accordo hanno due scelte davanti a loro: continuare a lavorare con le proprie teorie, finendo eventualmente nell'inattualità e nella marginalità; oppure adeguarsi all'opinione dominante. E non è certo una bella conclusione.

Per cercare di superare questo ostacolo occorre invece darsi un'ulteriore criterio. Si tratta del rapporto esistente tra i membri di una comunità scientifica per evitare che decisioni riguardanti la verità siano decise sulla base del potere di cui i membri di una comunità sono dotati. La domanda pertinente a questo riguarda è la seguente: in quale rapporto devono stare i membri di una comunità scientifica? La risposta è una e una sola. Perché questioni di verità siano decise sulla base di criteri scientifici, occorre che i membri della comunità siano in condizioni di parità di potere. Si deve a J. Habermas la migliore formulazione di questo problema e della sua soluzione. Secondo Habermas, i parlanti o, con il nostro linguaggio, i membri di una comunità scientifica devono essere in condizioni tali per cui:

1. Tutti i potenziali partecipanti ad un discorso debbono avere uguale possibilità di utilizzare atti linguistici comunicativi in modo tale che in ogni istante si possono aprire discorsi e continuarli mediante interventi e repliche, domande e risposte.

2. Tutti i partecipanti al discorso debbono avere la stessa possibilità di avanzare interpretazioni, affermazioni, raccomandazioni, spiegazioni e giustificazioni e di problematizzare, motivare o confutare le loro istanze di valore, cosicché nessuna opinione precostituita rimanga alla lunga sottratta alla tematizzazione e alla critica [...].

3. Sono ammessi al discorso solamente parlanti che in quanto persone agenti hanno le stesse chances di utilizzare atti linguistici rappresentativi, cioè di esprimere le loro disposizioni, sentimenti e intuizioni [...].

4. Sono ammessi al discorso solamente i parlanti che in quanto persone agenti hanno la stessa chance di utilizzare atti linguistici regolativi, cioè di comandare e opporsi, di permettere e di vietare, di fare promesse e di revocarle, di rendere conto e di pretendere di averne. (Habermas, 1973, pp. 337-338)

Habermas ha chiamato situazione discorsiva ideale questa condizione di simmetria di potere tra i parlanti. Solo in queste condizioni, tra due teorie, può prevalere quella che ha dalla sua parte «il migliore argomento» o, con il nostro linguaggio, le migliori ragioni logiche e sperimentali, individuate sulla base del criterio della coerenza della teoria e della sua corrispondenza con il mondo.

della luce furono tali che, anche se «La logica pura non lo costrinse affatto all'abbandono perché l'esperienza di Foucault non era affatto un experimentum crucis; tuttavia resistendo più a lungo all'ottica vibratoria, Biot avrebbe mancato di buon senso» (Duhem, 1914, p. 245). Come più tardi si dirà, la luce è sia ondulatoria, sia quantica. Per un'analisi della questione posta da Duhem cfr. Cotesta, 1999, pp. 134-147. 
Molte sono state le critiche avanzate verso la habermasiana situazione discorsiva ideale: soprattutto è stata criticata per la sua patente irrealtà. È vero, infatti, che nessuna delle comunità scientifiche opera in queste condizioni. Habermas, però, non la propone come una situazione empirica ma come un weberiano tipo ideale. Così intesa, la situazione discorsiva ideale serve a misurare il consenso vero e quello distorto da ragioni non scientifiche oppure, più modestamente, il grado di vicinanza/lontananza delle situazioni reali dalla situazione tipica.

Per riassumere, dunque, possiamo dire che il discorso o qualsiasi teoria che pretenda di essere considerata scientifica deve presentare i seguenti tre caratteri: a. Coerenza tra le proposizioni che compongono la teoria.

b. Corrispondenza della teoria con il mondo che vuole spiegare. La corrispondenza teoria/mondo può essere accertata dalla comunità scientifica mediante la teoria della verità come corrispondenza, lungo la linea Aristotele, Popper, Tarski, oppure mediante la teoria della verità come consenso.

c. Simmetria di potere tra i membri delle comunità scientifiche ${ }^{10}$.

\subsection{La base della distinzione tra le scienze e, in particolare, tra le scienze sociali}

Vediamo ora l'altra parte della luna: l'oggetto delle scienze e la loro distinzione. La nostra tesi su questo punto è che la differenza/distinzione tra le scienze si basa sull'oggetto da loro studiato. A questa definizione generica, però, dobbiamo aggiungere ulteriori specificazioni. Infatti, sia tra le scienze cosiddette naturali, sia tra quelle denominate sociali, lo stesso fenomeno può essere indagato da diversi punti di vista. Possiamo rendere chiara quest'affermazione con un esempio tratto dalla sociologia. Georg Simmel, nel definire l'oggetto della sociologia, così si esprime:

[...] quale può essere l'oggetto proprio e nuovo, la cui indagine fa della sociologia una scienza autonoma e dai confini determinati? È ovvio che per questa sua legittimazione quale scienza nuova non occorre la scoperta di un oggetto la cui esistenza fosse prima ignota. Tutto ciò che indichiamo in generale come oggetto è un complesso di determinazioni e di relazioni di cui ciascuna, proiettata su una pluralità di oggetti, può diventare oggetto di una scienza particolare. Ogni scienza poggia su un'astrazione, in quanto considera la totalità di una qual-

10 Il lettore che desiderasse avere più ampie informazioni su questa teoria della verità può consultare un mio vecchio libro Nuove legittimazioni (Cotesta, 1984) oppure il più recente Linguaggio e verità (Cotesta, 1999) nel quale la teoria è ripresa e giustificata con nuovi saggi epistemologici. 
che cosa, che non possiamo afferrare in modo unitario per mezzo di nessuna scienza, secondo uno dei suoi aspetti, cioè dal punto di vista di un determinato concetto. [...] La sociologia, nella sua relazione con le scienze esistenti, è quindi un nuovo metodo, uno strumento ausiliario della ricerca, per avvicinarsi ai fenomeni di tutti quei campi in modo nuovo. (Simmel, 1908, p. 7)

La sociologia, dice Simmel, non nasce perché si è scoperto un nuovo oggetto ma perché taluni fenomeni vengono guardati con uno sguardo nuovo. Questo sguardo è fondato da un'astrazione, da un concetto della cosa. Secondo la sua concezione della sociologia, lo sguardo nuovo consiste nel considerare la società fondata sulle azioni reciproche tra gli individui (Wechselwirkungen).

La sociologia, continua Simmel in un passo successivo,

[...] come scienza particolare potrebbe trovare il suo oggetto particolare soltanto tracciando una nuova linea attraverso certi fatti che, in quanto tali, sono perfettamente noti $[\ldots]$. Se deve dunque esserci una sociologia come scienza particolare, occorre pertanto che il concetto di società in quanto tale sottoponga i dati storico-sociali - al di là della raccolta estrinseca di quei fenomeni - a un nuovo processo di astrazione e di coordinamento, in modo tale che certe determinazioni degli stessi, prima considerate in altre e molteplici relazioni, vengano riconosciute come reciprocamente connesse e quindi come oggetti di un'unica scienza. (ivi, p. 8)

Per distinguere il proprio oggetto da quelli di altre scienze, eventualmente già ben consolidate come la storia e l'economia o in via di costituzione come la psicologia, la sociologia deve trattare fenomeni nuovi oppure già trattati da altre discipline, in modo nuovo. Questo modo nuovo, per Simmel, è considerare le azioni reciproche tra gli individui come capaci di esercitare o di subire «effetti»:

[La società] esiste là dove più individui entrano in azione reciproca. Quest'azione reciproca sorge sempre da determinati impulsi o in vista di determinati scopi. Impulsi erotici, religiosi o semplicemente socievoli, scopi di difesa e di attacco, di gioco e di acquisizione, di aiuto e di insegnamento, nonché innumerevoli altri, fanno sì che l'uomo entri con altri in una coesistenza, in un agire l'uno per l'altro, con l'altro e contro l'altro, in una correlazione di situazioni, ossia che eserciti effetti sugli altri e ne subisca dagli altri. (ivi, pp. 8-9)

Su questo punto si può trovare convergenza tra Simmel, Durkheim e Weber. Durkheim, infatti, ritiene che la sociologia consideri i fatti sociali come esterni agli individui e capaci di esercitare una certa costrizione su di loro.

Si potrebbe generalizzare questo discorso. Ogni scienza si costituisce come scienza particolare perché formula delle ipotesi sul suo oggetto. Per dirla in termini filosofici, la natura, l'universo o il mondo possono essere conside- 
rati come ordinati; e in questo caso il compito della scienza è quello di scoprire tale ordine; oppure essi possono essere considerati come un caos, il cui ordine è dovuto al caso; e in questo secondo caso le scienze cercano di scoprire come dal caos emerga un ordine; infine, il mondo può essere inteso come un caos e l'ordine come il risultato di una costruzione umana; e, in questo terzo caso, occorre vedere come procede la costruzione umana di un tale ordine.

La prima alternativa è piuttosto tradizionale e scontata. Le scienze moderne si fondano sull'idea che il mondo, nelle sue diverse accezioni, contenga un ordine implicito. È un'idea antica proposta dallo stoicismo e passata nelle società moderne occidentali. La seconda è in parte un lascito del mondo greco, propriamente del materialismo di Democrito. La terza è una concezione moderna.

Nella sociologia si impone, con la riflessione di Max Weber, la terza via. Il mondo è in sé puro caos; vi sono tuttavia delle generalizzazioni che possono dare senso ai casi singoli studiati dalla sociologia. Il senso, o se si vuole, l'ordine è una costruzione umana, sia sul versante storico, sia sul versante epistemologico e scientifico. A conclusione del suo saggio su La "oggettività» conoscitiva della scienza e della politica sociale (1904), Max Weber afferma:

[...] la vita nella sua realtà irrazionale ed il suo contenuto di possibili significati sono inesauribili, mentre la concreta configurazione della relazione di valore rimane fluida, sottoposta com'è al mutamento nell'oscuro avvenire della cultura umana. La luce, che emana da quelle supreme idee di valore, cade sempre su una parte finita, e continuamente mutevole, dellimmensa e caotica corrente degli avvenimenti che fluisce nel tempo. (Weber, 1904, p. 135; corsivo mio)

I due punti rilevanti del discorso weberiano sono appunto il fatto che (a) la realtà è inesauribile e caotica; (b) la luce che, sulla base delle supreme idee di valore, possiamo gettare su di essa è solo una parte finita di quella realtà e, tra l'altro, anch'essa mutevole, perché i valori, da cui parte la nostra «illuminazione» del mondo, sono mutevoli nel corso del tempo.

Se questa è la costituzione "metafisica» del mondo, come si fa a dargli un ordine? Senza entrare troppo nei dettagli del discorso weberiano possiamo procedere per gradi. Il primo consiste - e lo sappiamo già - nel riconoscere che questa realtà è esterna a noi. Di questa realtà noi ritagliamo una parte finita, di cui, nonostante quello che a livello di senso comune sappiamo di essa, vogliamo conoscere le connessioni tra le sue diverse dimensioni - la sua struttura - la sua genesi, il rapporto di causalità tra i suoi elementi ${ }^{11}$.

11 Anthony Giddens (1976) ha pensato questo rapporto tra senso comune ed analisi sociologica come un «doppio circuito ermeneutico»: dal senso comune all'analisi sociologica e, di nuovo, al senso comune che, a questo punto, ha una visione più corretta della realtà sociale. 
Il secondo passaggio consiste nella selezione di alcuni suoi caratteri «essenziali», avrebbe detto Durkheim, per costruire dei tipi puri, i famosi tipi ideali.

Il terzo passaggio consiste in un'operazione «fantastica» consistente nella introduzione di ipotesi controfattuali per comprendere se le relazioni causali tra gli elementi possano essere confermate oppure debbano essere abbandonate. «L'imputazione causale - afferma Weber - si compie nella forma di un processo concettuale, che implica una serie di astrazioni. La prima, e decisiva, è appunto quella che compiamo pensando una o alcune delle componenti causali oggettive del processo mutate in una determinata direzione e, chiedendoci se, nelle condizioni mutate dell'evento, sarebbe 'stata da aspettarsi' la medesima conseguenza (nei punti 'essenziali'), oppure un'altra» (Weber, 1906, p. 214). Per illustrare questa metodologia, diciamo, controfattuale del ragionamento scientifico, in dialogo critico con E. Meyer, Weber riprende una serie di esempi storici. Uno di questi, forse il più importante, è la discussione sulle conseguenze della battaglia di Maratona, di per sé un evento di scarsa rilevanza dal punto di visita militare. In realtà, la battaglia aveva come posta in gioco una "decisione' tra due 'possibilità - da un lato lo svolgimento di una cultura religioso-teocratica, i cui principi risiedono nei misteri e negli oracoli, sotto l'egida del protettorato persiano che ovunque utilizzava il più possibile la religione nazionale, come presso gli Ebrei, in quanto mezzo di dominio, e dall'altro la vittoria del libero mondo spirituale ellenico, orientato verso questo mondo, il quale ci donò quei valori culturali di cui ancor oggi ci nutriamo» (Weber, 1906, p. 214). Solo se introduciamo l'ipotesi controfattuale di una vittoria dei persiani nella battaglia di Maratona possiamo, da un lato, comprendere le connessioni causali che l'anno prodotta e, dall'altro, il suo significato per la «storia universale».

Il quarto passaggio consiste nel fatto che, una volta elaborata l'ipotesi controfattuale (terzo passaggio), occorre compararla con la realtà empirica. "Quale che sia il contenuto di un tipo ideale razionale - afferma Weber [...] la sua costruzione ha sempre, nell'ambito delle indagini empiriche, lo scopo soltanto di 'comparare' con esso la realtà empirica, e di stabilire il suo contrasto o la sua lontananza da essa oppure il suo relativo accostarsi ad essa, per poterla descrivere e intendere mediante l'attribuzione causale e quindi spiegarla, facendo uso di concetti intelligibili il più possibile univoci» (Weber, 1917, p. 366).

Come abbiamo detto sopra, il concetto di "causa» con cui lavora Weber già ai suoi tempi era criticato nelle scienze fisiche. Esso rinviava ad un «paradigma» delle scienze della natura, e in particolare della fisica, che proprio in quegli anni veniva scosso nelle sua fondamenta. Einstein, infatti, propose la sua Teoria della relatività ristretta nel 1905 e la sua Teoria della relatività 
generale nel 1916. I saggi metodologici di Weber risalgono al 1904, 1906, 1913, 1917. Nello stesso periodo egli lavorava ai saggi sulla sociologia della religione e all'ampio materiale poi confluito, a cura di sua moglie Marianne Weber, in Economia e società. Un ulteriore chiaro punto di contrasto riguarda l'ipotesi metafisica sul mondo: per Weber esso è un caos privo di ordine; per Einstein, invece esso ha un ordine che la fisica deve cercare di scoprire, se è vera la sua battuta rivolta contro Niels Bohr secondo cui «Dio non gioca a dadi» ${ }^{12}$. Su un altro punto invece si ritrova convergenza: nel modo di intendere la verità e in un atteggiamento anti-relativistico. Nel saggio $L a$ "oggettività» conoscitiva della scienza e della politica sociale (1904) Weber affronta in diversi passaggi la questione della verità (Weber, 1904, pp. 66, 67, $72,100)$. Poiché potrebbe sembrare che i suoi riferimenti alle idee di valore, quale criterio per stabilire quale parte finita della realtà infinita considerare rilevante ed oggetto della scienza, potrebbe condurre ad un relativismo conoscitivo e alla incomunicabilità tra ricercatori, Weber ritorna più volte sul punto. Il riferimento ai valori è determinante per individuare quale parte della realtà è rilevante per una scienza. Ma, come poi dice nel saggio sull' «avalutatività», il riferimento ai valori non può sostituire l'onere della prova razionale nell'argomentazione scientifica. Nel saggio suddetto, scritto per il gruppo dell'Archiv für Sozialwissenschaft und Sozialpolitik, di cui costituisce un documento programmatico, riassumendo le posizioni già disseminate nel corso del testo, scrive:

[Le] idee di valore sono, fuori di ogni questione, «soggettive». Tra l'interesse «storico» per una cronaca di famiglia e quelle per lo sviluppo dei più grandi fenomeni di cultura [...] c'è un'infinita gradazione di «significati» [...]. E tuttavia esse sono invero storicamente mutevoli con il carattere della cultura e dei principi che guidano gli uomini. Da ciò non discende ovviamente che la ricerca delle scienze della cultura possa dar luogo soltanto a prodotti i quali siano «soggettivi» nel senso che valgono per l'uno e non per l'altro. Ciò che cambia è piuttosto il grado in cui essi interessano l'uno e l'altro. In altri termini, ciò che diventa oggetto dell'indagine, ed in quale misura questa si estenda nell'infinità delle connessioni causali, è determinato soltanto dalle idee di valore che dirigono il ricercatore e la sua epoca; [...] nel metodo della ricerca [...] il «punto di vista» a cui si ispira è determinante per l'elaborazione degli strumenti concettuali che egli impiega - mentre nel modo della loro applicazione il ricercatore è di certo, qui come ovunque, vincolato alle norme del nostro pensiero. Poiché verità scientifica è soltanto ciò che esige di valere per tutti quelli che vogliono la verità. (Weber, 1904, p. 100)

12 In verità, Einstein avrebbe detto: «Sembra difficile dare una sbirciata alle carte di Dio. Ma che Egli giochi a dadi e usi metodi 'telepatici' [...] è qualcosa a cui non posso credere nemmeno per un attimo" (cit. in Bryson, 2008). 
Vi sono, dice Weber, delle norme logiche «universali» a cui debbono attenersi tutti quelli che cercano la verità. In due passi precedenti dello stesso testo aveva detto: «[...] una dimostrazione scientifica corretta nel campo delle scienze sociali, condotta in forma metodica, deve essere riconosciuta come giusta, allorché essa abbia realmente conseguito il suo scopo, anche da un cinese»(Weber, 1904, p. 66) e "una rivista - l'Archiv - di scienza sociale nel senso da noi illustrato, in quanto aspira al carattere di scienza, deve essere una sede nella quale si cerca la verità, ed una verità tale - per rimanere nell'esempio - che esiga anche per il cinese la validità propria di un ordinamento concettuale della realtà empirica» (ivi, p. 67).

Crediamo a questo punto di potere riassumere. Se mettiamo insieme le argomentazioni sviluppate nel paragrafo 3.1 e quelle del paragrafo 3.2, abbiamo la struttura di un "paradigma scientifico». Il discorso fatto per la sociologia può essere fatto per altre scienze: economia, psicologia, storia, fisica e così via. Non si può tuttavia negare che intorno al concetto di «paradigma» - e fin dalla sua prima formulazione, ci sono - e ci sono stati - problemi. Lo stesso Thomas Kuhn, che lo ha introdotto nella storia della scienza, ha difficoltà a darne una definizione. Anzi, secondo Margareth Masterman (1970) nel testo di Kuhn, La struttura delle rivoluzioni scientifiche, vi sarebbero ben ventuno, se non di più, accezioni. La Masterman dimostra analiticamente ciò che altri critici di Kuhn avevano precedentemente osservato. Tanto che Kuhn, nel "Proscritto» alla seconda edizione del volume (1969) è costretto a cercare di dare una definizione del concetto di paradigma. Secondo la formulazione di Kuhn (1969, pp. 220-226) il paradigma è una "matrice disciplinaria» composta di quattro elementi: (a) una generalizzazione simbolica; (b) «paradigmi metafisici» o "parti metafisiche dei paradigmi», dogmi condivisi dai membri di una comunità scientifica come credenze in particolari modelli euristici o ontologici; (c) valori condivisi come coerenza interna o esterna, elegan$\mathrm{za}$, utilità sociale della scienza; (d) esempi comuni di soluzione di problemi. $\mathrm{Si}$ potrebbe forse riassumere questa complessa definizione dicendo che un paradigma è una struttura di (a) teorie, (b) modelli, (c) criteri condivisa da una comunità scientifica. La teoria delle rivoluzioni scientifiche proposta da Kuhn ha dato luogo a contestazioni e sviluppi di cui qui non mi posso occupare. Un punto soltanto, però, può essere utile trattare: la questione della traducibilità, dell'incomunicabilità e dell'incomparabilità tra paradigmi.

Secondo Kuhn i paradigmi sono traducibili l'uno nell'altro (Kuhn 1969 , p. 244). La traducibilità, tuttavia, non implica che il paradigma venga accettato da coloro che lo traducono nel proprio. Rimangono ad avviso di Kuhn diversità che non possono essere comprese dalle comunità costituite intorno ad una visione del mondo, intorno ad un paradigma scientifico. Per questo, anche, Kuhn è stato accusato di relativismo culturale e scientifico. 
Forse una via per risolvere questo problema consiste nella distinzione tra i diversi livelli o componenti di un paradigma scientifico. Se ci si pone a livello delle assunzioni metafisiche o ontologiche riguardanti il mondo o, più nello specifico, la realtà intono a cui si costituisce una scienza, la tesi di Kuhn ha buone ragioni dalla sua parte. Se invece ci si colloca a livello della logica della ricerca, la possibilità di comunicare e di intendersi tra comunità scientifiche è reale. La stessa "conversione» da un paradigma all'altro, proposta da Kuhn, non sarebbe possibile se non potesse essere legittimata da argomentazioni logiche condivise tra le comunità scientifiche o tra i loro membri. Nelle scienze sociali questo significa che vi possono essere diversi paradigmi distinti sulla base delle assunzioni di base - le ipotesi ontologiche sul mondo sociale - ma convergenti nell'uso dell'unica logica scientifica possibile. Ed è a questo livello della logica dell'argomentazione scientifica che i risultati prodotti dall'una o dall'altra indagine condotta a partire dalle assunzioni dei rispettivi paradigmi vengono valutate, accettate e rifiutate.

Un esempio tratto dalla storia e dalla sociologia contemporanee può aiutarci ad illustrare questo concetto. Da alcuni decenni è in corso una «rivoluzione» nelle scienze sociali ${ }^{13}$. Con minore o maggiore enfasi, molti affermano che l'era del «nazionalismo epistemologico» è finita. Cosa significa tutto questo? È cambiata la logica del ragionamento scientifico? No. E cambiata, sta cambiando, l'unità analitica di base della ricerca. La «nazione» è sostituita dalla "società globale». Anche se con eccessiva semplificazione, si ritiene che la sociologia del XX secolo abbia svolto i suoi discorsi nell'ambito dello statonazione. Il sistema sociale, insomma, e la società sarebbero stati considerati coestensivi dell'organizzazione politica.

Sebbene la costruzione della società globale sia un processo iniziato almeno cinque secoli orsono, nelle scienze sociali la revisione è cominciata solo da alcuni decenni ${ }^{14}$. La nuova assunzione di base è che la «società» è la formazione umana composta da tutti gli uomini e le donne del mondo. Le loro distinzioni particolari sono appunto componenti di questo insieme. E se vogliamo capire i processi delle diverse aree del mondo, occorre inserirli in questo orizzonte globale. La stessa costruzione degli stati-nazione si può comprendere meglio a partire dal contesto globale (Conrad, Eckert, \& Freitag, 2007).

Ciò che è cambiato - o sta lentamente cambiando - nelle scienze sociali, è solo la dimensione dell'unità analitica, non, ad esempio, le ipotesi

13 Per avere un'idea del cambiamento in corso nelle scienze sociali sia consentito un riferimento a due mei lavori: Cotesta, 2004 e 2008.

14 Si devono richiamare almeno i tentativi di Toynbee, Braudel, C. Schmitt, Wallerstein, Elias. Per tutto ciò cfr. Cotesta, 2004 e 2008. 
metafisiche o ontologiche su di essa. Ognuno può continuare a credere, ed a cercare, nel mondo le leggi che ne reggono l'ordine, oppure la costruzione di un ordine che si impone al caso. Sull'assunzione fondamentale che considera la società come "globale» si possono inserire altre caratteristiche ed usarle come distinzione/differenza tra paradigmi.

\section{RIFERIMENTI BIBLIOGRAFICI}

Adorno, T. W., Popper, K., et al. (1969). Der Positivismusstreit in der deutschen Soziologie. Neuwied - Berlin: Luchterland Verlag (trad. it., Dialettica e positivismo in sociologia. Torino: Einaudi, 1972).

Aristotele (1994). Metafisica. Roma - Bari: Laterza.

Berthelot, J.-M. (Ed.). (2001). Épistémologie des sciences sociales. Paris: PUF.

Bianco, F. (1978). Il dibattito sullo storicismo. Bologna: Il Mulino.

Boudon, R. (1994). Durkheim e Weber: convergence de méthode. In M. Hirschhorn \& J. Coenen-Huter (Eds.), Durkheim, Weber vers la fin des malentendus. Paris: L'Harmattan.

Bryson, B. (2008). Breve storia di (quasi) tutto. Milano: TEA.

Cavalli, A. (2012). Momenti di storia del pensiero sociologico. Milano: LED.

Conrad, S., Eckert, A., \& Freitag, U. (Hg). (2007). Globalgeschichte, Frankurt am Main: Campus Verlag.

Cotesta, V. (1984). Nuove legittimazioni. Roma: Ianua.

Cotesta, V. (1999). Linguaggio e verità. Roma: Armando.

Cotesta, V. (2004). Sociologia del mondo globale. Roma - Bari: Laterza.

Cotesta, V. (2008). From nation-state to global society: The changing paradigm of contemporary sociology, International Review of Sociology, 18(1), 19-30.

Duhem, P. (1914). La théorie physique. Son object, sa structure. Paris: Rivière (trad. it., La teoria fisica. Bologna: Il Mulino, 1978).

Durkheim, É. (1895). Les règles de la méthode sociologique. Paris: Alcan (trad. it., Le regole del metodo sociologico. Torino: Einaudi, 2008).

Eisenstadt, S. N. (1997). Modernità, modernizzazione e oltre. Roma: Armando.

Eisenstadt, S. N. (2003). Comparative civilizations and multiple modernities, Voll. 1-2. Leiden: Brill.

Erodoto (2000). Storie. Milano: Rizzoli.

Giddens, A. (1976). New rules of sociological method: A positive critique of interpretative sociologies. London: Hutchison (trad. it., Nuove regole del metodo sociologico. Bologna: Il Mulino, 1979). 
Goody, J. (1996). The East in the West. Cambridge: Cambridge University Press (trad. it., L'Oriente in Occidente. Bologna: Il Mulino, 1999).

Goody, J. (2006). Europe and Islam. In G. Delanty (Ed.), Europe and Asia beyond East and West. London: Routledge.

Habermas, J. (1973). Auszug aus «Wahrheitstheorien». In AA.VV., Wirklichkeit und Reflexionen. Festschrift für Walter Scultz. Pfullingen: Neske (trad. it., Discorso e verità. In J. Habermas, Agire comunicativo e logica delle scienze sociali, pp. 319343. Bologna: Il Mulino, 1980).

Joyce, J. (1973). Ulisse. Milano: Mondadori.

Kuhn, T. S. (1962, 1970). The structure of scientific revolutions. Chicago, IL: University of Chicago Press (trad. it., La struttura delle rivoluzioni scientifiche. Torino: Einaudi, 1969 e 1978; in questa edizione è pubblicato il «Proscritto» del 1969).

Masterman, M. (1970). La natura di un paradigma. In I. Lakatos \& A. Musgrave (Eds.), Criticism and the growth of knowledge. Cambridge: Cambridge University Press (trad. it., Critica e crescita della conoscenza. Milano: Feltrinelli, 1993).

Popper, K. R. (1934). Logik der Forschung. Wien: Julius Verlag (trad. ingl., The logic of scientific discovery. London: Hutchinson, 1959; trad. it., Logica della scoperta scientifica. Torino: Einaudi, 1970).

Popper, K. R. (1969a). Conjectures and refutations. London: Routledge \& Kegan Paul (trad. it., Congetture e confutazioni. Bologna: Il Mulino, 1972).

Popper, K. R. (1969b). Verità, razionalità e accrescersi della conoscenza scientifica. In Id., Conjectures and refutations (pp. 369-428). London: Routledge \& Kegan Paul.

Schutz, A. (1971). Collected papers. The Hague: Martinus Nijhoff (trad. it. a cura di A. Izzo, Saggi sociologici. Torino: UTET, 1979).

Simmel, G. (1908). Sociologie. Berlin: Dunker \& Humblot (trad. it., Sociologia. Milano: Edizioni di Comunità, 1980).

Smelser, N. J. (1976). Comparative methods in the social sciences. Englewood Cliffs, NJ: Prentice-Hall (trad. it., La comparazione nelle scienze sociali. Bologna: Il Mulino, 1982).

Tarski, A. (1943-1944). The semantic conception of truth. Philosophy and Phenomenological Research, 4 (trad. it. in AA.VV., Semantica e filosofia del linguaggio. Milano: Il Saggiatore, 1969).

Tucidide (1998). La guerra del Peloponneso. Milano: Rizzoli.

Weber, M. (1904). L'«oggettività» conoscitiva della scienza sociale e della politica sociale. In Weber, 1922, 1951.

Weber, M. (1906). Studi critici intorno alla logica delle scienze della cultura. In Weber, 1922, 1951.

Weber, M. (1913). Alcune categorie della sociologia comprendente. In Weber, 1922 , 1951. 
Weber, M. (1917). Il significato della «avalutatività» delle scienze sociologiche e economiche. In Weber, 1922, 1951.

Weber. M. (1922). Gesammelte Aufsätze zur Religionssoziologie. Tübingen: J.B.C. Mohr (trad.it. a cura di P. Rossi, Sociologia della religione. Milano: Edizioni di Comunità, 2002).

Weber, M. (1922, 1951). Gesammelte Aufsätze zur Wissenschaftslehre. Tübingen: J.B.C. Mohr (trad. it. a cura di P. Rossi, Il metodo delle scienze storico-sociale. Torino: Einaudi, 1958; Milano: Mondadori, 1974. Le citazioni del testo provengono dall'edizione Mondadori).

Weber M. (1922, 1956). Wirtschaft und Gesellschaft. Tübingen: J.B.C. Mohr (trad. it. a cura di P. Rossi, Economia e società. Milano: Edizioni di Comunità, 1980).

\section{RiassUNTO}

L'autore sostiene la tesi secondo la quale il "New Realism» è una salutare reazione contro gli aspetti più deleteri del post-modernismo. Nello stesso tempo però sostiene che il richiamo alla realtà e al realismo sia un aspetto ricorrente nel dibattito scientifico, sia nei periodi di fondazione di nuove scienze, sia nei periodi di cambiamenti di paradigma. Questa tesi è dimostrata mediante l'analisi di due casi: la critica di Tucidide verso Erodoto al momento della fondazione della storia come scienza e il brusco richiamo alla realtà fatto da Durkheim nelle sue "Regole del metodo sociologico". La tesi dell'autore però è che questo richiamo alla realtà è accompagnato da una nuova visione del mondo sociale. E questo si vede meglio nelle opere metodologiche di $M$. Weber. Attraverso lo studio di questi casi l'autore propone la sua tesi principale secondo la quale le scienze - tutte le scienze empiriche e non solo le scienze sociali - sono tali se, e solo se, la loro struttura logica è fondata su tre caratteri: coerenza tra i suoi principi teorici; corrispondenza della teoria con il mondo (di cui si pone come spiegazione scientifica); libertà, autonomia e responsabilità dei ricercatori. Tutte le scienze - e non solo le scienze empiriche - si distinguono invece l'una dall'altra per il loro oggetto elo per il modo in cui intendono il loro oggetto. Questa tesi generale è illustrata mediante lo studio del caso delle scienze sociali e, in particolare, della struttura epistemologica della sociologia.

Parole chiave: Durkheim, Metodologia, Paradigmi, Scienze sociali, Weber. 